\title{
SPECT/CT in hyperparathyroidism
}

\author{
Angela Spanu • Orazio Schillaci • Bastiana Piras • \\ Giuseppe Madeddu
}

Received: 7 September 2014 / Accepted: 14 November 2014/Published online: 27 November 2014

(C) Italian Association of Nuclear Medicine and Molecular Imaging 2014

\begin{abstract}
Hybrid SPECT/CT imaging is playing an increasingly important role in nuclear medicine in several oncological and non-oncological fields. This review presents a literature survey on the use of SPECT/CT as an adjunct to parathyroid scintigraphy in patients with hyperparathyroidism (HPT), highlighting the certainties and the controversies that have so far emerged in this regard. SPECT/CT, providing both functional and anatomical data, has proved to be superior to conventional planar parathyroid scintigraphy and to SPECT for the precise localization and characterization of areas of marked uptake of radiotracers (MIBI or tetrofosmin) corresponding to abnormal parathyroid glands, thus facilitating their surgical treatment. The major benefits of SPECT/CT have been observed in patients with ectopic adenomas, mainly in the mediastinum, and in those with previous neck surgery. In some studies, SPECT/ $\mathrm{CT}$ has also demonstrated a higher sensitivity than conventional planar or SPECT scans particularly in the detection of low-weight parathyroid adenomas and in the presence of concomitant nodular goiter. In other studies, SPECT/CT has also been found to be more specific, with the
\end{abstract}

Color figures online at http://link.springer.com/article/10.1007/ s40336-014-0089-4

A. Spanu $(\bowtie) \cdot$ B. Piras · G. Madeddu

Section of Nuclear Medicine, Department of Clinical and Experimental Medicine, University of Sassari, 07100 Sassari, Italy

e-mail: angela.spanu@email.it

O. Schillaci

Department of Biomedicine and Prevention, University of Rome

Tor Vergata, 00133 Rome, Italy

O. Schillaci

IRCCS Neuromed Pozzilli, 86077 Pozzilli, Isernia, Italy anatomical data provided by the CT component shown to be of help in correctly categorizing MIBI-avid non-parathyroid lesions. No definitive conclusions on the clinical value of SPECT/CT in patients with secondary HPT and multiglandular disease can be done due to the small number of studies thus far conducted, but the current available data nevertheless seem to suggest that hybrid imaging is able to detect a significantly higher number of hyperplastic glands than planar scanning can.

Keywords Hyperparathyroidism · Parathyroid scintigraphy $\cdot$ SPECT $\cdot$ SPECT/CT $\cdot$ MIBI

\section{Introduction}

Hyperparathyroidism (HPT) is an endocrine disorder due to increased activity of the parathyroid glands that have the function of maintaining calcium and phosphate homeostasis [1].

The parathyroid glands, or parathyroids, are small ellipsoid-shaped structures normally located behind the thyroid glands in close proximity to the upper and lower poles of both thyroid lobes. Although it is usual to have four parathyroid glands, approximately $10 \%$ of individuals may have more (between five and seven) and 2-3\% have fewer than four. Moreover, in almost $4-16 \%$ of cases, parathyroids (more often inferior parathyroids) can be present in ectopic sites. Depending on their embryonic development, it is therefore possible to find superior parathyroid glands, which originate from the 4th branchial arch, in the carotid sheath, within the thyroid, or in the tracheoesophageal groove of either the neck or the superior mediastinum. Inferior parathyroid glands, which, like the thymus, originate from the 3 rd branchial arch, may be 
located below the lower pole of the thyroid lobe, either in the thyrothymic ligament or within the cervical portion of the thymus, within the thyroid, along the carotid sheath, or in the anterior mediastinum, in this latter case being either within the thymus or in contact with the great vessels.

There are essentially three types of HPT: primary (p), secondary (s), and tertiary (t) HPT [1].

pHPT is a relatively common disease, affecting approximately $0.7 \%$ of the general population in Western countries [1, 2]. It is due to the autonomous overproduction of parathyroid hormone (PTH) by one or more parathyroid glands, leading to inappropriately elevated intact PTH serum levels and hypercalcemia. The most frequent cause of pHPT is a single parathyroid adenoma $(80-85 \%$ of cases); more rarely, it is caused by multiglandular disease (MGD), either double adenoma (5\%) or glandular hyperplasia (10-15\%), while parathyroid carcinoma is a very rare cause $(<1 \%)$. Parathyroid hyperplasia of one or more glands is particularly frequent in patients with hereditary forms of pHPT (familial HPT, multiple endocrine neoplasia syndromes, HPT-jaw tumor syndrome).

sHPT is generally a complication of chronic renal failure, but it can also be associated with vitamin D deficiency or vitamin $\mathrm{D}$ resistance, intestinal absorption abnormalities, and renal leak syndrome; all these conditions may cause hypocalcemia which represents the stimulus for PTH hypersecretion, leading to generalized hyperplasia of the parathyroid glands $[1,3]$.

In long-standing renal disease, and usually after kidney transplantation, the parathyroid glands may continue to grow and diffuse hyperplasia may develop into asymmetric nodular hypertrophy becoming autonomous and leading to hypercalcemia. This condition is known as tHPT $[1,3]$.

In patients with pHPT and hypercalcemia, the definitive cure is surgical resection of the hyperfunctioning parathyroid gland [2]. Bilateral neck exploration with examination of all four parathyroid glands has been the standard of care for many years. However, considering that a single parathyroid adenoma is the most frequent cause of pHPT, more recent years have seen an increasing interest in minimally invasive parathyroidectomy, in which the surgical resection is limited solely to the hyperfunctioning gland, obviating the need for bilateral neck exploration. Minimally invasive parathyroidectomy, which may even be performed endoscopically, requires accurate preoperative imaging studies to identify and localize the adenoma and may benefit from the combined use of intraoperative PTH assay to confirm adenoma removal, thereby improving the chances of achieving a complete surgical cure.

In sHPT, the medical treatment represents the first-line therapeutic option, but when the disease becomes resistant to medical therapy, parathyroidectomy, either subtotal parathyroidectomy or total parathyroidectomy with autotransplantation, should be considered [3]. Parathyroidectomy may also be necessary in tHPT in patients with refractory hypercalcemia.

However, in both pHPT and sHPT, the initial surgery may not provide a definitive cure due to: failure to identify ectopic lesions, the presence of unsuspected MGD or autonomous hyperplasia of the parathyroid autografts, or insufficiently extensive surgery in known MGD, leading to persistent or recurrent disease that requires a second operation. In pHPT patients, HPT may persist after parathyroidectomy in 2-22\% of cases and recur in $1-10 \%$ of cases [4], whereas in SHPT patients persistent disease has been observed in 5-10\% of cases and recurrent disease in $20-30 \%$ of cases [5].

\section{Parathyroid scintigraphy}

Role and indications

Parathyroid imaging has no role in the diagnosis of HPT, which is based on biochemical and clinical data; thus, a negative parathyroid scan does not exclude a diagnosis of HPT.

Instead, parathyroid imaging plays a very important role in the preoperative identification of hyperfunctioning parathyroid gland(s) in HPT patients who are candidates for surgery and in helping the surgeon to find the most appropriate approach, thus reducing operating times and limiting complications and surgical failure.

At present, ${ }^{99 \mathrm{~m}} \mathrm{Tc}$-sestaMIBI (MIBI) parathyroid scintigraphy is recognized as the imaging procedure of choice in this field and it is especially indicated in patients with pHPT at their first operation who are scheduled to undergo a minimally invasive parathyroidectomy [6, 7]. In such patients, it is suggested to use the technique in combination with high-resolution neck ultrasound (US) in order to further increase the sensitivity and accuracy of detection and localization of parathyroid adenomas located in the neck. Moreover, the addition of US is particularly indicated in patients with concomitant thyroid nodules for better differentiating these from parathyroid lesions. Thin-section computed tomography (CT) and MRI may also be useful as additional imaging modalities in the presence of ectopic mediastinal parathyroid adenomas since they allow detailed anatomical localization [6, 7].

The role of preoperative parathyroid scintigraphy in patients with sHPT, who are in any case candidates for bilateral neck exploration, remains controversial due to the low sensitivity demonstrated by scintigraphy in the identification of hyperplastic glands [6,7].

Preoperative MIBI parathyroid scintigraphy is, instead, mandatory in patients with persistent or recurrent disease because of the increased likelihood of supernumerary 
parathyroid glands and ectopic localization and the increased risk of complications and surgical failure in the presence of distortion and scarring caused by previous surgical interventions $[6,7]$. Selective venous sampling of parathyroid hormone has been considered the gold standard in this setting, but it has certain limitations: it is invasive, operator dependent, and requires considerable experience to achieve successful results [8].

MIBI parathyroid scintigraphy, finally, is a prerequisite for the intraoperative localization of hyperfunctioning parathyroid tissue using a gamma-probe or a small camera (radioguided minimally invasive parathyroidectomy) [6, 7].

\section{Acquisition protocol}

For parathyroid scintigraphy, two-dimensional planar scanning continues to be the standard acquisition method, performed according to either the dual-tracer subtraction or the single-tracer dual-phase protocol $[6,7]$.

Necessitated by the absence of radiotracers that are trapped exclusively in parathyroid tissue, the dual-tracer subtraction protocol rests on the use of two different radiotracers, one that is exclusively taken up by thyroid tissue $\left({ }^{99 \mathrm{~m}}\right.$ Tc-pertechnetate or $\left.{ }^{123} \mathrm{I}\right)$ and another that is taken up by both thyroid and parathyroid tissue $\left({ }^{99 \mathrm{~m}} \mathrm{Tc}-\mathrm{MIBI}\right)$. After acquisition, the thyroid image is subtracted from the thyroid/parathyroid one, leading to the visualization of the hyperfunctioning parathyroid tissue only. For thyroid imaging, radioiodine should be preferred to ${ }^{99 \mathrm{~m}} \mathrm{Tc}$-pertechnetate since a radioiodine thyroid image and a MIBI thyroid/parathyroid image may be acquired simultaneously, avoiding the problem of artifacts on the subtraction image. However, ${ }^{123} \mathrm{I}$ is more expensive than ${ }^{99 \mathrm{~m}} \mathrm{Tc}$-pertechnetate and requires a longer interval between injection and image acquisition. ${ }^{99 \mathrm{~m}}$ Tc-tetrofosmin can be used as an alternative to MIBI in the dual-tracer protocol, since it, too, accumulates in both thyroid and parathyroid tissue.

Instead, the single-tracer dual-phase protocol is based on the differential washout of MIBI from thyroid and parathyroid tissue, with washout from thyroid glands usually being faster than that from hyperfunctioning parathyroid glands. This protocol thus requires the acquisition of two sets of images after the i.v. injection of MIBI: early (15 min) and delayed (2-3 h) images; hyperfunctioning parathyroid tissue usually appears as a focal area of increased uptake in the early images that becomes more evident on the delayed images. However, this is not always the case, given that the retention of MIBI in parathyroid tissue is affected by several biological factors; parathyroid adenomas thus present variable washout kinetics, and many hyperplastic parathyroid glands show a faster washout. Moreover, some thyroid nodules, such as adenomas and carcinomas, present the same uptake pattern usually seen in parathyroid adenomas. The combination with a thyroid scintigraphy and/or with a thyroid US may help in differentiating thyroid from parathyroid nodules. Tetrofosmin is not suitable for the dual-phase protocol since it is not characterized by differential washout in the thyroid and parathyroid glands.

Irrespective of the acquisition protocol applied, parathyroid planar MIBI imaging involves the acquisition of at least two images: one, acquired with a parallel-hole collimator, that includes both the entire neck and the thorax (from the angle of the jaw to the upper part of the myocardium) in the field of view to ensure the visualization of ectopic and supranumerary parathyroid glands, and the other focused on the neck and magnified in the thyroid/parathyroid area; for this latter image, as well as for the thyroid image in the dualtracer protocol, the use of a pinhole collimator is suggested for optimal resolution and greater sensitivity. Additional lateral or anterior-oblique views of the neck may be useful in the presence of a focal area of increased MIBI uptake adjacent to the inferior pole of the thyroid gland to help in differentiating between an inferior adenoma (anterior position, close to the thyroid) and a superior adenoma that has migrated caudally in the tracheoesophageal groove or behind the esophagus (posterior position).

Standard planar parathyroid scintigraphy, performed either with the dual-tracer or with the single-tracer dualphase technique, has proved very effective in the detection of parathyroid adenomas in pHPT patients, achieving sensitivity values equal or superior to $90 \%$ in most series, whereas its sensitivity in detecting hyperplastic glands in sHPT patients appears much lower [5, 9].

\section{Single-photon emission tomography (SPECT)}

In the last two decades, SPECT has been increasingly employed in parathyroid imaging as a complementary tool to conventional planar imaging. SPECT data should be acquired over a $360^{\circ}$ arch, including the neck and the thorax (from the angle of the jaw to the upper part of the myocardium) in the field of view, using a body-contoured elliptic orbit, obtaining 120 projections at 15-25 s/per projection (every $3-6^{\circ}$ angle), and using a matrix of $128 \times 128$ and suitable zoom factor [6, 7].

SPECT may be included in both dual-tracer and singletracer dual-phase protocols, and in the latter it may be acquired in the early or in the delayed phase, or in both. However, SPECT acquired in the early phase should be preferred to delayed SPECT as it gives a lower number of false-negative results related to hyperfunctioning parathyroid glands with rapid washout, as demonstrated by PerezMonte et al. in a series of 47 patients: 33 at their first surgical exploration and 14 who were candidates for repeat 
surgery because of persistent or recurrent HPT [10]. In this series, early SPECT gave $91 \%$ sensitivity for detection and localization of adenomas, whereas the sensitivity of delayed SPECT was $74 \%$ for detection and $32 \%$ for localization, with a statistically significant difference for both localization $(P<0.001)$ and detection $(P=0.03)$.

When compared with standard planar scanning, SPECT offers certain intrinsic advantages. The multidimensional and maximum intensity projection (MIP) images provided by SPECT are more accurate in defining the depth of hyperfunctioning parathyroid lesions in relation to the thyroid gland and when these lesions are present within the mediastinum.

The major advantages of SPECT over planar scanning have been demonstrated, in particular, in patients affected by $\mathrm{pHPT}$, with SPECT found to show higher sensitivity especially in the detection of small, deeply located and ectopic parathyroid adenomas and in the presence of concomitant multinodular goiter (MNG) [11, 12].

Moka et al. [11], in a series of 55 patients with pHPT scheduled for bilateral neck exploration, combined early planar MIBI imaging with delayed SPECT; multiplanar SPECT and MIP images increased planar sensitivity from 71 to $95 \%$, showing a higher performance especially in the detection of small adenomas $\leq 500 \mathrm{mg}$, only $56 \%$ of which were positive on planar MIBI imaging, while $91 \%$ of these were positive on SPECT. SPECT also showed a higher sensitivity in larger adenomas, but the difference was less marked (97 versus $81 \%$ ).

Lorberboym et al. [12] added early SPECT to standard MIBI planar dual-phase and dual-tracer (using pertechnetate for thyroid imaging) scanning in a consecutive series of 52 patients with pHPT who were candidates for minimally invasive surgery. Early SPECT increased planar sensitivity from 79 to $96 \%$, proving to be superior mainly in patients with ectopic adenomas or with concomitant MNG. In the same series, washout kinetics of MIBI proved unreliable in the planar dual-phase technique, delayed MIBI retention being present in only $60 \%$ of adenomas.

Gallowitsch et al. [13] compared tetrofosmin/pertechnetate subtraction planar scintigraphy with SPECT in a series of 23 patients with suspected parathyroid adenoma or hyperplasia due to pHPT or tHPT in an endemic goiter area. In this series, no difference between planar and SPECT in the detection of parathyroid adenomas was found, with both imaging modalities showing $87 \%$ sensitivity; however, the combined use of the two procedures increased the sensitivity to $97.5 \%$.

Staudenherz et al., adding delayed SPECT to MIBI dualphase planar scanning in a series of 56 pHPT patients with a high prevalence of concomitant thyroid diseases, also failed to find differences in sensitivity between SPECT and planar images, although SPECT gave additional information regarding the exact topographical localization of the parathyroid tumor in patients with superimposed thyroid disease or previous neck surgery [14].

These advantages of SPECT, which make it a better guide for the surgeon, especially in patients with ectopic cervico-mediastinal posterior adenomas, have also been reported by Billotey et al. in a mixed population of 43 patients with HPT (24 with pHPT, 16 with sHPT, and 3 with tHPT), including 33 with persistent or recurrent HPT [15]. For each patient, dynamic scintigraphy, planar, and SPECT images of the neck and thorax were acquired. The authors analyzed the kinetics of tracer uptake in the thyroid area using specific software with factor analysis of dynamic structures.

In contrast with the data obtained in pHPT patients, and especially in those with parathyroid adenomas, the superiority of SPECT over planar scanning in the detection of hyperplastic glands and in SHPT patients remains debatable.

Civelek et al. assessed the feasibility of delayed SPECT, as a single stand-alone test, in a very large series of patients with pHPT (338 patients: 287 unexplored and 51 reexplored); 400 abnormal glands were excised at surgery, including 299 single adenomas, 23 double adenomas, and 55 hyperplastic glands. Sensitivity was high in adenomas, both single and double (96 and $83 \%$, respectively), but low in hyperplastic glands, only $45 \%$ of which were true positive [16].

Gallowitsch et al. compared tetrofosmin planar and SPECT scintigraphy in 48 patients, 35 with pHPT and 13 with sHPT; SPECT increased planar sensitivity in both pHPT and sHPT (from 69.2 to $94.4 \%$ and from 38 to $61.5 \%$, respectively), but the value reached in the latter patients with hyperplastic glands appeared unsatisfactory [17].

Better results have been obtained by Neumann et al. using ${ }^{123}$ I/MIBI subtraction SPECT which showed $77 \%$ sensitivity in the identification of hyperplastic glands in 19 patients with chronic renal failure and sHPT [18]. The same authors in another study, carried out in a selected series of patients with sHPT who had previously undergone total parathyroidectomy and autotransplantation, obtained no false-negative results in localizing hyperplastic parathyroid tissue [19]. All of the parathyroid autotransplants in the sternocleidomastoid muscle were successfully identified in this series of patients. Given that these lesions are difficult to localize preoperatively, even using high-resolution morphological procedures such as CT, the authors concluded that ${ }^{123} \mathrm{I} / \mathrm{MIBI}$ subtraction SPECT can be considered the imaging procedure of choice for the preoperative localization of abnormal parathyroid tissue in patients with postoperative recurrent sHPT.

Spanu et al. tested a pinhole collimator instead of a parallel-hole collimator for SPECT, limiting the 
examination to the neck due to the small field of view of the pinhole. Pinhole SPECT, due to its very high spatial resolution, proved more sensitive than planar parathyroid scintigraphy especially in the detection of small and deeply located adenomas as well as in the identification of hyperplastic glands in patients with sHPT [20, 21]. However, pinhole SPECT cannot be used routinely since it requires software specifically designed for image acquisition and processing, and the new gamma cameras are not supplied with such software.

\section{SPECT/CT}

As stated above, knowledge of the precise localization of hyperfunctioning parathyroid glands in patients with HPT scheduled for surgery is crucial in order to achieve a definitive cure. Adenomas located anteriorly in the neck are preferably treated through a mini anterior incision, whereas those located posteriorly may be removed through a lateral approach. At the same time, in the mediastinum, accurate preoperative localization may direct the surgical approach, leading the surgeon to opt for a median sternotomy or a left or right thoracotomy.

Although SPECT, compared with two-dimensional planar scanning, allows better lesion localization, it lacks anatomical landmarks. The addition of CT to SPECT (SPECT/CT) and the fusion of anatomical (CT) and functional (SPECT) images can further improve the topographic assignment of lesions including their relationship to the neighboring structures (e.g., the thyroid gland, trachea, esophagus, and vessels). CT and SPECT images can be acquired with two separate devices and then fused using dedicated software or, preferably, anatomical and functional images can be acquired sequentially (with the patient remaining in the same imaging bed) using a hybrid SPECT/ CT device which integrates a SPECT camera with a CT scanner, thus avoiding co-registration errors.

Like SPECT alone, SPECT/CT may also be included in both dual-tracer and single-tracer dual-phase protocols. If planar images are acquired, they should be obtained first [7]. In the MIBI dual-phase protocol, SPECT/CT may be acquired in the early or in the delayed phase, or in both. However, considering that $\mathrm{CT}$ acquisition exposes patients to radiation each time it is performed, one should consider carefully whether CT should be used at both time points or at only a single time point [7].

The acquisition parameters of the SPECT component of SPECT/CT are the same as those mentioned above for SPECT alone. With regard to the CT component, the optimal slice thickness, acquisition time, and CT parameters ( $\mathrm{mAs}$ and $\mathrm{kVp}$ ) should be determined by individual laboratories or suggested by the manufacturer and should aim to maximize image quality and to minimize radiation exposure to the patient. Low-dose CT is generally employed, with a tube current of $2.5 \mathrm{mAs}$ and a voltage of $140 \mathrm{kVp}$ (range: 100-140 kVp). Higher tube current values may be used, thus giving diagnostic CT. Intravenous contrast enhancement is not usually used, but it may be useful or justifiable in selected cases [7].

CT images can be used not only as anatomical landmarks but also for attenuation correction. The use of attenuation-corrected (AC) SPECT images rather than non$\mathrm{AC}$ images has been suggested by some authors, since AC images seem to facilitate the identification of adenomas, especially within the mediastinum, due to their better image contrast [22].

This review presents a literature survey on the use of SPECT/CT as an adjunct to parathyroid scintigraphy in patients with HPT. A comprehensive computer literature search of "PubMed/MEDLINE" was conducted to find published original articles on the diagnostic performance of SPECT/CT in HPT. We used a search algorithm based on the combination of the terms "SPECT/CT" and "hyperparathyroidism." No start date limit was used; the search was updated to August 30, 2014. No language restriction was used.

The use of MIBI SPECT and image-fused CT was first reported in the early 2000s in two case reports in which SPECT/CT allowed precise preoperative localization of two ectopic mediastinal parathyroid adenomas, directing the successful excision of the hyperfunctioning lesions via minimally invasive surgery. In both studies, SPECT and CT data were acquired with two separate SPECT and CT devices and then fused by using dedicated fusion software $[23,24]$.

The usefulness of MIBI SPECT/CT for the correct preoperative localization of ectopic mediastinal parathyroid adenomas was then confirmed by Kaczirek et al. [25] in a series of four patients, two with pHPT and two with persistent SHPT, in whom SPECT and CT were acquired by means of a hybrid device.

Table 1 summarizes, in chronological order, the most important studies (at least ten patients per study) in the current literature reporting the clinical role of SPECT/CT in patients affected by HPT. The parathyroid scintigraphic protocols and the main SPECT and CT acquisition parameters employed by the different authors are also reported. The SPECT/CT studies were generally performed in comparison with conventional imaging methods (planar scanning, SPECT, US, or CT).

SPECT/CT in patients with $\mathrm{pHPT}$

Gayed et al. [26] evaluated the additional value of MIBI SPECT/CT, acquired in the early phase, over conventional 


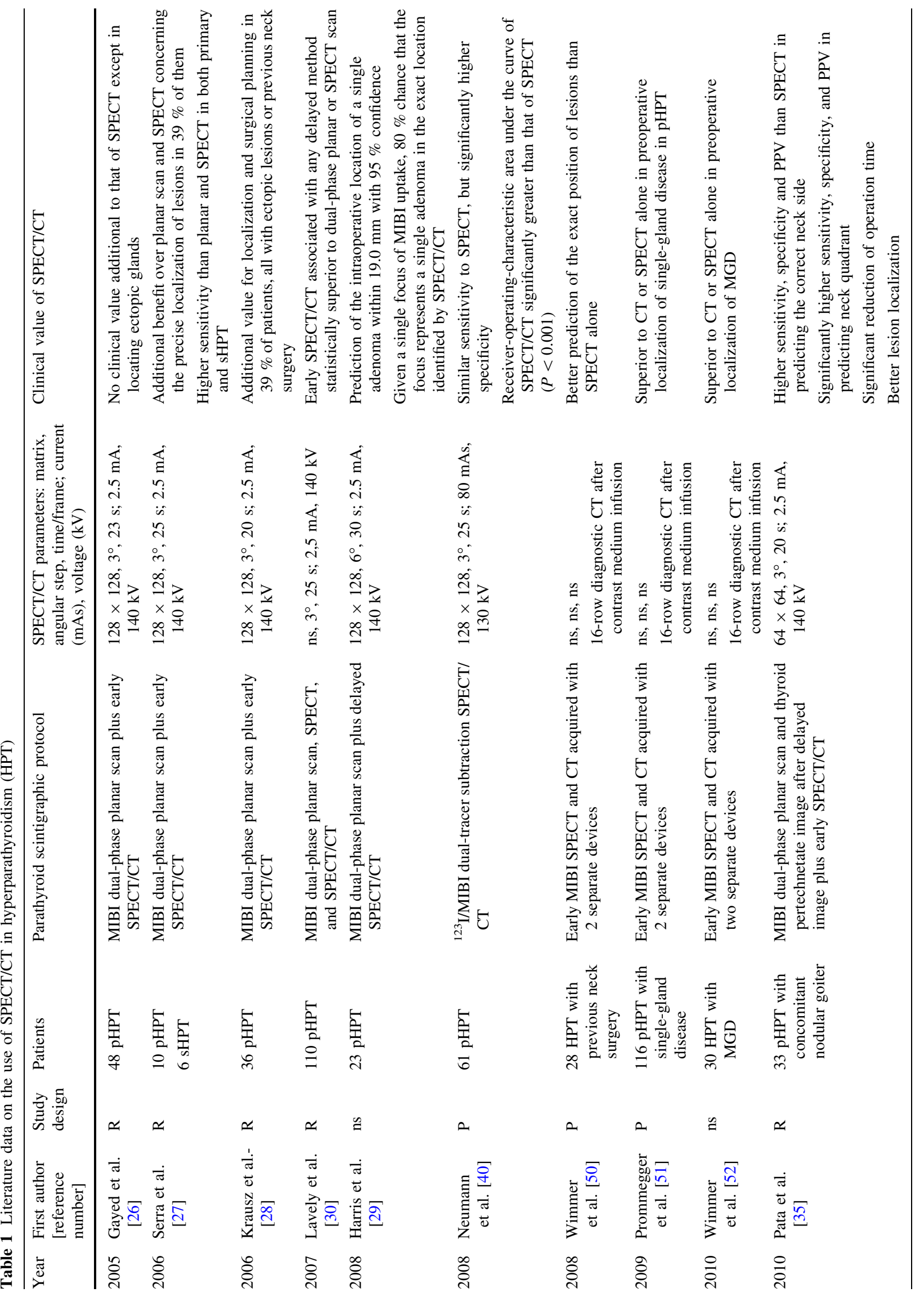




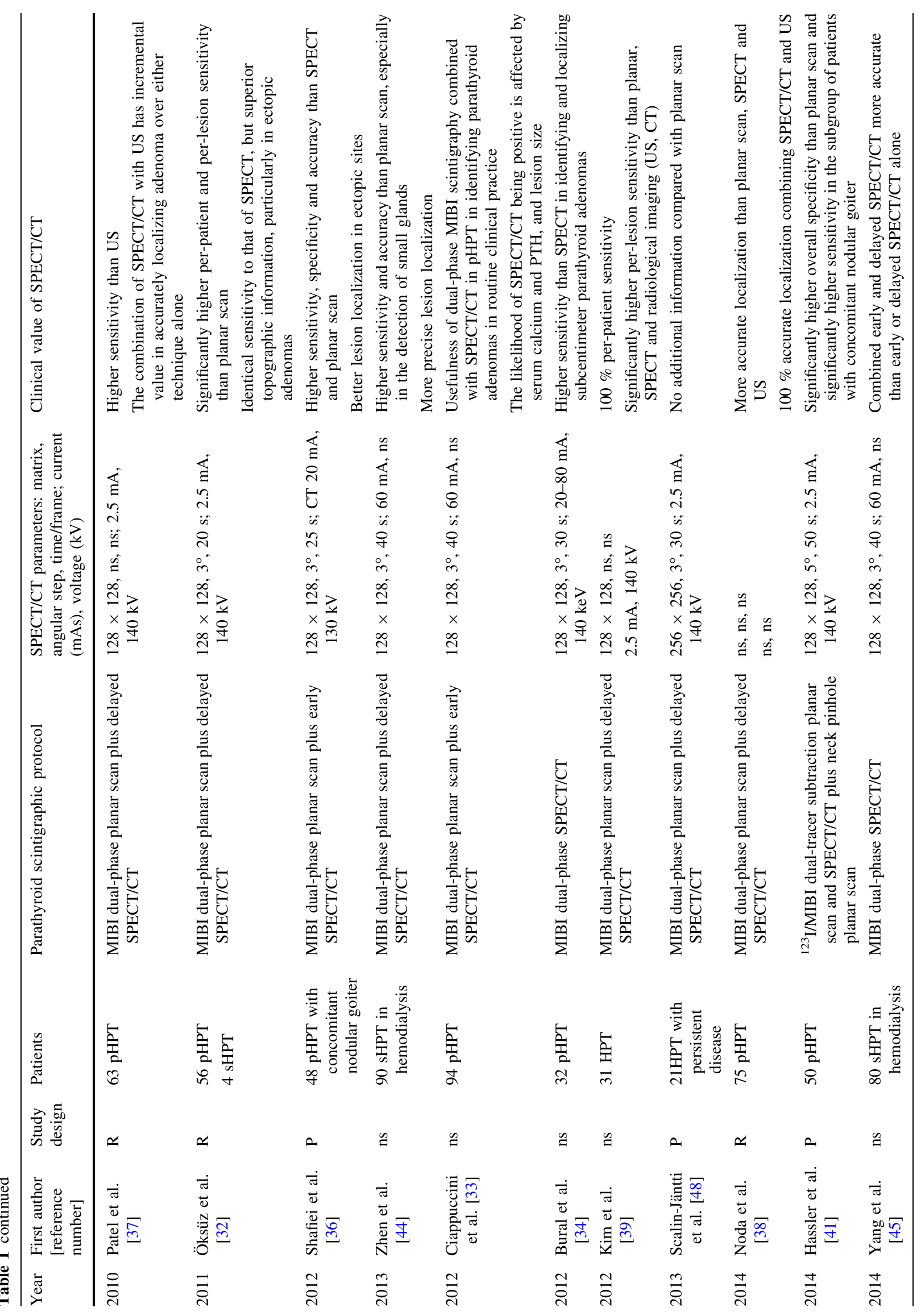


dual-phase planar and SPECT scans in diagnosing and locating both parathyroid adenoma and parathyroid hyperplasia in a series of 48 patients with pHPT. SPECT/ CT changed the diagnosis, from positive to negative, in only one patient $(2 \%)$ and better located the glands in only four patients $(8 \%)$, proving particularly helpful in locating the two ectopic parathyroid adenomas. The authors concluded that SPECT/CT does not add significant clinical value to that of conventional SPECT except in locating ectopic parathyroid glands.

SPECT and SPECT/CT sensitivities in adenoma or hyperplastic gland detection have also been compared in other studies summarized in Table 2, while Table 3 reports the studies in which the clinical impact of SPECT/CT in ectopic lesion detection was assessed.

Serra et al. [27] applied the same acquisition protocol utilized by Gayed et al. in a selected series of 16 patients (most of whom were affected by pHPT) with 23 MIBIpositive glands (16 hyperplastic glands, 5 adenomatous glands, 1 parathyroid carcinoma, and 1 thyroid carcinoma), 14 in ectopic sites and nine in normotopic sites. The patients also underwent neck US which was inconclusive in 12/16 patients affected by MNG, but identified two probable ectopic parathyroid glands. The inclusion criteria were: HPT with a clinical indication for surgery and glands not clearly identified by US due to concomitant presence of MNG, HPT with US negative for parathyroid localization in patients with normal thyroid gland, HPT in patients with ectopic glands detected by planar parathyroid scintigraphy. SPECT/CT gave a higher sensitivity than planar scanning (100 versus $57 \%$ in pHPT patients and 64 versus $43 \%$ in sHPT patients) and the same sensitivity as SPECT. However, fusion imaging showed additional benefit over planar dual-phase scanning and SPECT in the precise localization of lesions, simplifying resection in $39 \%$ of lesions, and radically changing the surgical approach in three patients with parathyroid glands located in the retrotracheal space.

Krausz et al. [28] also added early SPECT/CT to planar dual-phase scanning in a selected series of patients $(n=36)$ with pHPT with a negative planar scan or with an ill-defined focus in the neck or an ectopic site on planar imaging. The incremental value of SPECT/CT with regard to lesion localization and the surgical procedure was assessed. In this series, SPECT/CT contributed to localization and surgical planning in 14/36 (39\%) patients: ten with ectopic parathyroid adenomas and four with cervical adenomas who had distorted neck anatomy following previous failed parathyroidectomy (3/4 cases) or total thyroidectomy ( $1 / 4$ cases). In particular, the results changed the surgical planning in six of the ten patients with ectopic adenomas in whom sternotomy was avoided as SPECT/CT allowed precise anatomical localization of the lesions at the level of the suprasternal notch and 
manubrium. In this series, SPECT/CT was of limited additional value in the detection of cervical adenomas in patients undergoing initial surgery. Moreover, SPECT/CT did not increase the sensitivity of planar scanning, with both procedures detecting $92 \%$ of adenomas subsequently confirmed at surgery.

Figure 1 illustrates the case of a pHPT patient with an ectopic mediastinal parathyroid adenoma precisely localized by SPECT/CT.

Harris et al. [29] also assessed the accuracy of MIBI SPECT/CT (acquired at $2 \mathrm{~h}$ ), added to planar dual-phase scintigraphy, in predicting the precise intraoperative anatomical location of parathyroid adenomas, using both qualitative and quantitative analyses; for the latter, they measured the distance between the location of the adenoma on SPECT/CT and the location of the adenoma pinpointed intraoperatively. In a series of 23 patients, SPECT/CT correctly detected and localized 16 of 18 cases of single adenomas, predicting the intraoperative location of a single parathyroid adenoma within $19.0 \mathrm{~mm}$ with $95 \%$ confidence. The median interval from incision to identification of the adenoma was $14 \mathrm{~min}$ (range: 8-40 min). Moreover, the authors demonstrated that, in the setting of pHPT, a single focus of MIBI uptake has an $80 \%(95 \% \mathrm{CI}$ 97.4-66.5\%) chance of corresponding to a single parathyroid adenoma in the exact location indicated by the SPECT/CT scan. On the contrary, the correct detection and localization of multiple glands remained very difficult since only one of two cases of double adenomas was identified, and none of the three cases of multiglandular hyperplasia.

Lavely et al. [30] studied a very large series $(n=110)$ of patients with pHPT and no prior surgery, 98 of whom had single adenomas and were the subject of their study. Six image sets (early and delayed planar, SPECT, and SPECT/CT images) were acquired and compared with each other, also to determine whether dual-phase imaging is advantageous for these three methods. Surgical location served as the standard. In this study, SPECT/CT allowed the highest reader confidence in localizing parathyroid adenomas. Moreover, early SPECT/CT in combination with any delayed imaging method (SPECT/CT, SPECT, or planar imaging) was statistically superior to dual-phase planar imaging or dual-phase SPECT. Moreover, for all three methods (planar, SPECT, and SPECT/CT), dualphase imaging was superior to single-phase imaging. The authors concluded that dual-phase imaging protocols, that must include early SPECT/CT, should be included in the routine preoperative evaluation of patients with $\mathrm{pHPT}$ scheduled for surgery, underlining that the major advantage of SPECT/CT seems to be its ability to differentiate inferior from inferoposterior glands (superior glands in the tracheoesophageal groove).
A pHPT case with an inferior parathyroid adenoma is illustrated in Fig. 2.

Tokmak et al. [31] also acquired and compared six image sets (early and delayed planar, SPECT, and SPECT/ CT images) in a series of patients $(n=154)$ with pHPT before neck surgery; they also considered the definitive histology reports (168 lesions: 140 single eutopic adenomas, 4 double adenomas, 4 ectopic adenomas, and 16 hyperplastic glands in MEN-1 patients). In this study, too, the combination of early-phase SPECT/CT with any delayed imaging method proved to be the best diagnostic protocol in terms of both sensitivity and positive predictive value. Moreover, in this series, SPECT/CT proved more sensitive than planar scanning (per-patient sensitivity: 97.9 versus $87.7 \%$ ), especially in low-weight adenomas, identifying $28 \%$ more lesions than planar scanning and also detecting more hyperplastic glands, regardless of their size. In particular, in patients with hyperplastic glands (MEN-1 patients), SPECT/CT revealed about $7 \%$ more lesions than planar imaging did. The authors also demonstrated that the addition of neck US to SPECT/CT did not significantly affect the correct preoperative localization rate $(P=0.30)$.

Öksüz et al. [32] assessed the accuracy of parathyroid imaging for the detection of parathyroid adenoma and glandular hyperplasia comparing dual-phase planar scanning, SPECT, and SPECT/CT in a series of 60 patients, 56 with pHPT and four with SHPT. SPECT/CT demonstrated a significantly higher per-patient and per-lesion sensitivity than planar scanning did (97 and $95 \%$ versus 76 and $69 \%$, respectively), due to the superior contrast resolution, lesion depth information, and accurate three-dimensional localization, all factors contributing to increase the detection rate of particularly small adenomas. SPECT/CT had identical sensitivity compared to SPECT alone, but it provided superior topographic information, particularly in patients with ectopic adenomas. In 8/60 patients, C-11 methionine PET or PET/CT was also performed, resulting positive in all cases (per-patient sensitivity: $100 \%$ ) and identifying $8 / 9$ ( $89 \%$ ) lesions; the authors thus recommended the use of this latter procedure, as an additional diagnostic tool, in cases with negative MIBI findings and proven HPT.

Ciappuccini et al. [33], in their single-institution experience, assessed the diagnostic value of SPECT/CT in pHPT patients in a consecutive series of 94 patients and analyzed the relationship between SPECT/CT data and serum calcium or PTH concentrations. Fifty-nine studies $(63 \%)$ were positive, demonstrating a single focus in 56 cases and double foci in three. Serum calcium and PTH levels, as well as adenoma size, affect the likelihood of SPECT/CT being positive, serum biological markers, and lesion size being significantly higher $(P<0.001)$ in patients with a positive scintigraphy than in those with a negative scan. Moreover, in patients with a measurable 
parathyroid adenoma on integrated CT scan $(n=43)$, the greatest axial diameter of the adenoma was correlated with serum calcium or PTH levels. Fifty-four patients underwent surgery. Pathological glands were ascertained at surgery in $48 / 54$ patients (a single adenoma in 46 cases, one parathyroid carcinoma in one case, and a double adenoma in one case). SPECT/CT was true positive in $44 / 48$ cases and true negative in five of the remaining six patients without abnormal glands, resulting in a sensitivity of $92 \%$ and a specificity of $83 \%$.

Bural et al. [34] demonstrated the greater benefit of SPECT/CT over SPECT alone especially for the accurate detection and localization of subcentimeter parathyroid adenomas. In a series of 32 patients with elevated PTH levels (29 with one surgically proven adenoma and three with hyperplastic glands), SPECT correctly identified and localized the hyperfunctioning parathyroid glands in 22 cases $(69 \%)$, while SPECT/CT did so in 31 cases (97\%). All the parathyroid lesions not detected with SPECT in ten patients were less than $10 \mathrm{~mm}$ in size. The low sensitivity of SPECT in the detection of subcentimeter lesions was explained by the authors as an effect of the poor spatial localization ability of SPECT alone, i.e., the absence of a precisely registered CT limits the detection of small lesions. Also the only false-negative patient on SPECT/CT had a small adenoma $(8 \mathrm{~mm})$.

Other authors have focused on the use of SPECT/CT in patients with pHPT and concomitant thyroid nodules.

Pata el al. [35] assessed the clinical performance of MIBI SPECT/CT compared to conventional SPECT in a selected series of 33 patients with pHPT and concomitant nodular goiter to determine whether SPECT/CT is more useful than SPECT in guiding the surgeon during parathyroidectomy, particularly minimally invasive parathyroidectomy. SPECT/CT gave higher sensitivity, specificity, and positive predictive values than SPECT (93.7, 92.9, and $93.7 \%$ versus $80,87.5$, and $88.9 \%$, respectively) in predicting the correct neck side of abnormal parathyroid glands, although the differences were not statistically significant. The sensitivity and positive predictive values of SPECT/CT were significantly higher than those of SPECT ( 87.5 versus $55.6 \%, P<0.0001$, and 87.5 versus $62.5 \%$, $P<0.0022)$ in correctly identifying the neck quadrant affected by pHPT. Moreover, as for the relationship between imaging and operating time, the authors observed a slight, but significant, reduction of operating time after evaluation with SPECT/CT. In particular, SPECT/CT correctly identified the position of the adenoma in relation to the trachea, esophagus, and spine and thus optimized the surgical procedure.

Shafiei et al. [36] also assessed the usefulness of MIBI scintigraphy in a series of 48 patients with pHPT and concomitant nodular goiter scheduled for surgery, comparing early SPECT and SPECT/CT with dual-phase planar scanning. SPECT/CT gave higher sensitivity, specificity, and accuracy values than both SPECT and planar scanning (the corresponding values were $77.5,96.77$, and $85 \%$ for SPECT/CT versus $67.34,87.9$, and $75 \%$ for SPECT, and $59.18,77.41$, and $66.25 \%$ for planar scanning, respectively). There were nine sites that were found to be better localized on SPECT/CT scans than on SPECT images; five of these were located in the ectopic regions (thyrothymic ligament, tracheoesophageal groove, and thymus).

Some authors have assessed the efficacy of MIBI SPECT/CT, in comparison with high-resolution neck US, for planning minimally invasive parathyroidectomy in patients with pHPT $[37,38]$.

Patel et al. [37] retrospectively evaluated 63 patients with pHPT, 59 of whom had a solitary adenoma at surgery. US preoperatively identified 35/59 adenomas (64\%), whereas SPECT/CT identified 53/59 (90\%). Combined ultrasound and SPECT/CT identified 56 solitary parathyroid adenomas with concordant results in 35/56 cases (59\%), while three cases were positive only on US and 18 only on SPECT/CT; the adenoma was deep-seated or ectopically located in 14 of these latter cases, and there was co-existing nodular thyroid disease in eight cases. Combined US and SPECT/CT had an overall sensitivity of $95 \%$ and an overall accuracy of $91 \%$ for the preoperative localization (superior or inferior) of solitary parathyroid adenomas. There was no statistically significant difference in gland localization using SPECT/CT alone compared with SPECT/CT and US $(P>0.05)$. The authors concluded that the combination of US and SPECT/CT has incremental value in accurately localizing parathyroid adenomas compared with each technique alone and allows the selection of patients with pHPT who would be eligible for minimally invasive surgery. These conclusions are in agreement with those of Noda et al. [38] who retrospectively evaluated $75 \mathrm{pHPT}$ patients with a surgically proven single parathyroid adenoma. In this latter study, which also evaluated the clinical effectiveness of MIBI planar scanning, the rates of accurate identification of the laterality of lesions (left or right) by US, planar scanning, and SPECT/ CT were 83.6, 85.2, and 88.5\%, respectively. The corresponding values for precise lesion localization (right upper, right lower, left upper, left lower area of the thyroid gland or ectopic site) were 77.0, 75.4, and $88.5 \%$ of the evaluable cases, respectively. Precise localization was achieved in $90.5 \%$ of cases when combining US with planar scanning and in $100 \%$ when combining US with SPECT/CT. In this study, the superior sensitivity of SPECT/CT over US was confirmed in ectopic glands, $100 \%$ of which were found to be positive on fusion imaging.

Kim et al. [39] assessed the efficacy of MIBI SPECT/CT for minimally invasive parathyroidectomy comparing 


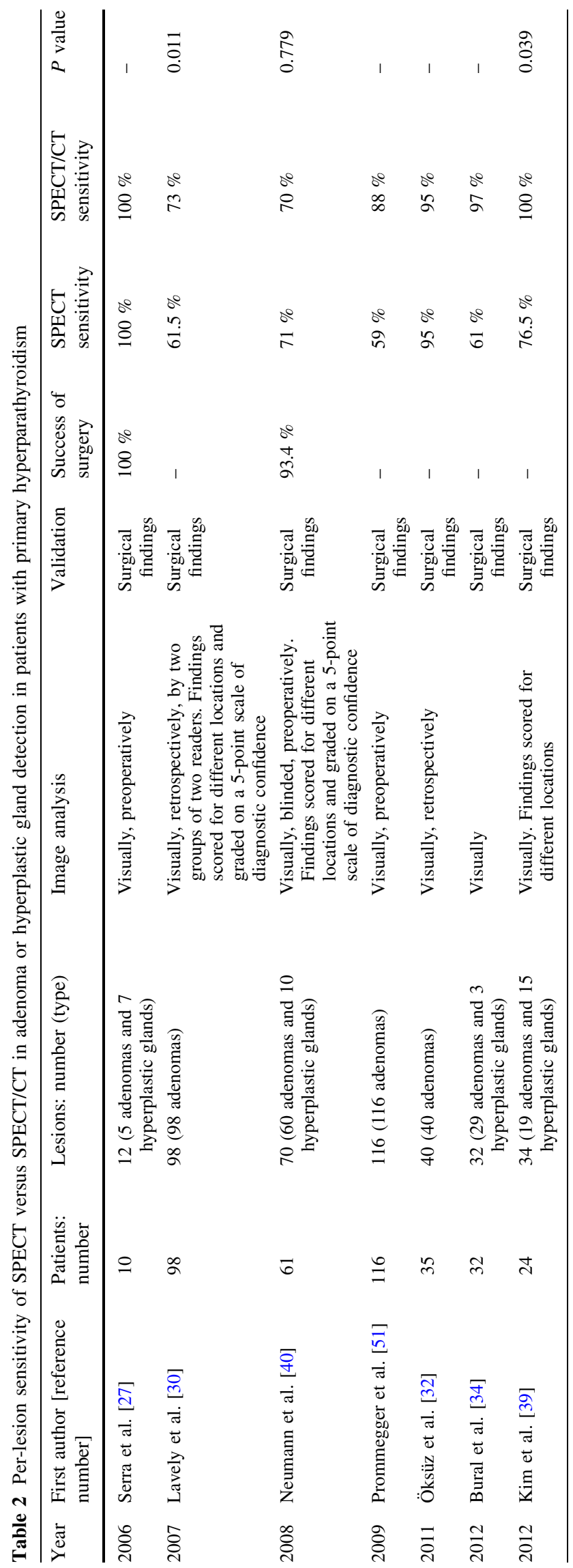

delayed SPECT/CT to dual-phase planar scanning, SPECT, and conventional imaging (US and CT) in a series of 31 patients with HPT; 24/31 were positive on scintigraphy and underwent surgery, leading to surgically proven adenomas in 19/24 cases and the finding of parathyroid hyperplasia in 5/24 (total: 35 lesions). SPECT/CT demonstrated $100 \%$ sensitivity in a patient-based analysis and significantly higher pre-lesion sensitivity when compared with all the other imaging procedures, correctly identifying the precise location of lesions. The final clinical diagnosis in seven patients with normal SPECT/CT was secondary hyperparathyroidism at 6 months of follow-up.

Neumann et al. [40] included SPECT/CT in the dualtracer $\mathrm{MIBI} /{ }^{123} \mathrm{I}$ subtraction parathyroid protocol. The authors compared the diagnostic accuracy of $\mathrm{MIBI} /{ }^{123} \mathrm{I}$ subtraction SPECT with SPECT/CT for the localization of abnormal parathyroid glands in a series of 61 patients with pHPT. Surgery was successful in 57/61 cases. SPECT and SPECT/CT showed similar sensitivity values (71 versus $70 \%, P>0.05)$, but the specificity of SPECT/CT was considerably higher than that of SPECT (89 versus $48 \%$, $P<0.05)$ because of the ability of SPECT/CT to better characterize SPECT findings based on their corresponding CT findings. In 13 cases, this ability allowed correct categorization of SPECT findings either as MIBI-avid nonparathyroid lesions (thyroid nodules) or as artifacts (longus colli muscle), thus reducing the number of false-positive findings. The authors also correlated SPECT and SPECT/ CT detectability with lesion weight, serum calcium, and serum PTH. The calcium and PTH levels were similar in true-positive and false-negative cases for both modalities, whereas the weights of the lesions differed between the true positives and false negatives, the weights for the true positives tending to be higher, particularly for SPECT/CT, in this latter case reaching statistical significance $(P=0.023)$. Finally, the authors demonstrated that the receiver-operating-characteristic area under the curve of SPECT/CT was significantly greater than that of SPECT $(P<0.001)$.

SPECT/CT dual-tracer MIBI/ ${ }^{123}$ I parathyroid scintigraphy was also tested by Hassler et al. [41] in comparison with pinhole planar scanning. Each parathyroid uptake was allocated to a neck parathyroid site (left superior, left inferior, right superior, right inferior) or classed as ectopic. The authors studied a series of 50 patients with sporadic pHPT who underwent planar scanning (neck and mediastinum parallel-hole images plus anterior neck pinhole images) and neck and mediastinum SPECT/CT, all with the subtraction method. The sensitivity in locating parathyroid adenomas was equivalent for the two protocols in the patients overall (86\% for SPECT/CT and $75 \%$ for planar scanning, $P=\mathrm{ns}$ ), but SPECT/CT gave a significantly higher specificity (100 versus $90 \%, P<0.04)$ allowing 


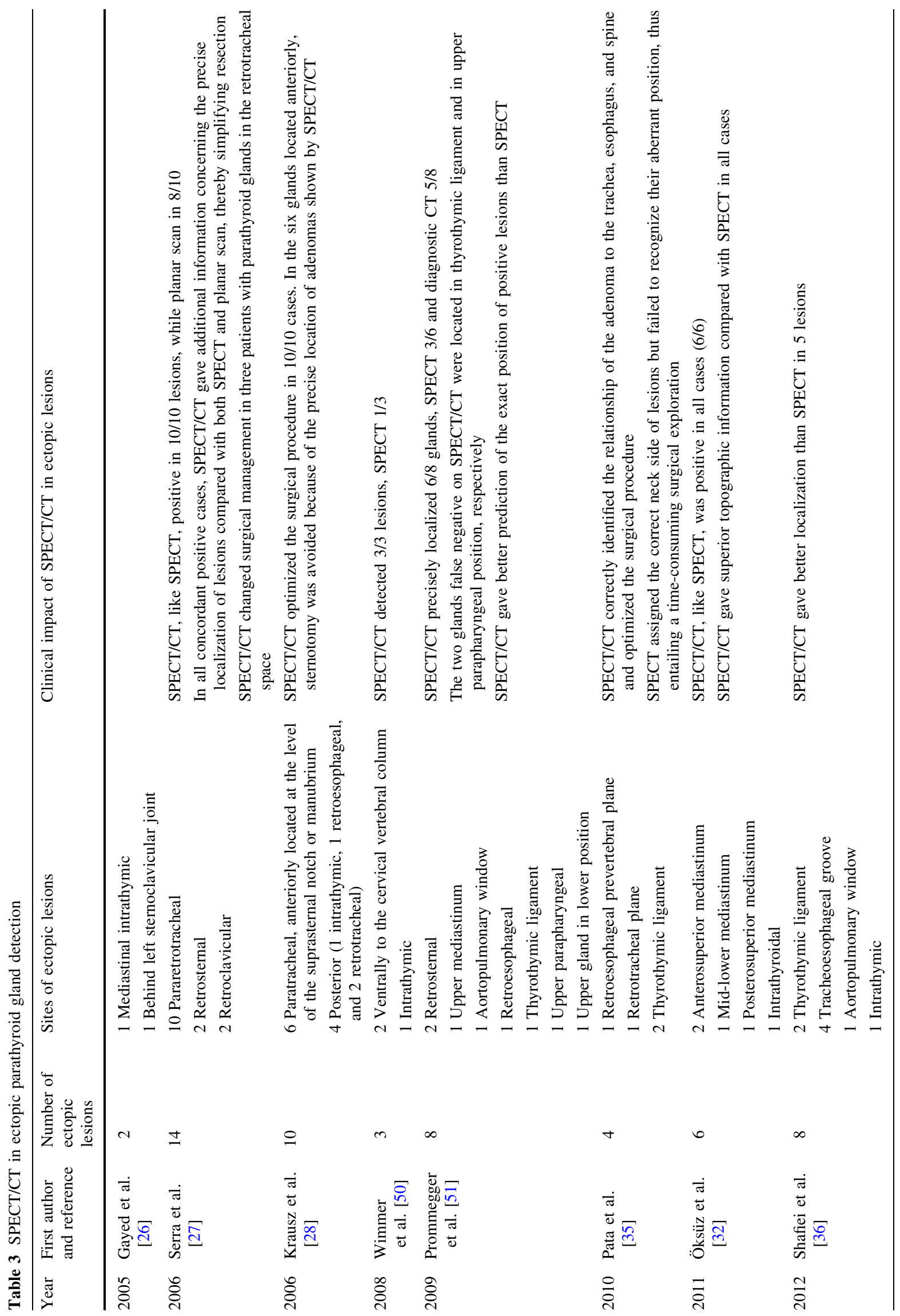


accurate anatomical localization for the surgeon. The sensitivity of SPECT/CT became significantly higher than that of planar scanning ( 88 versus $62 \%, P<0.04$ ) in the subgroup of patients with concomitant nodular goiter, but the specificity was similar (100 versus $95 \%, P=\mathrm{ns})$. In patients without thyroid disease, the sensitivity obtained with the two protocols was exactly the same $(85 \%$ in both cases). On the basis of the above results, the authors proposed that, for the purpose of obtaining preoperative assessments of pHPT patients and for guiding surgery, SPECT/CT subtraction should be performed first and the examination should then be completed using a neck pinhole collimator only if tomoscintigraphy is negative.

\section{SPECT/CT in patients with sHPT}

The literature reports only a few studies that specifically focused on patients affected by sHPT [42-45].

Citawa et al. [42] described a single case of a patient with advanced sHPT submitted to parathyroidectomy following parathyroid scintigraphy. In this case, SPECT/CT revealed all four involved glands, one more than MIBI dual-phase planar scanning did, and, thanks to the detailed information it gave regarding the relationship of the lesions to anatomical landmarks (which neither SPECT alone nor planar imaging provides), it allowed their rapid intraoperative localization.

Figure 3 illustrates the case of one sHPT patient with recurrent disease who had two ectopic hyperplastic parathyroid glands precisely located by SPECT/CT.

Chroustova et al. [43] assessed the effectiveness of early and delayed MIBI SPECT/CT and 3D subtraction ${ }^{99 \mathrm{~m}} \mathrm{Tc}-$ pertechnetate SPECT in a series of 61 patients with sHPT due to chronic renal disease. MIBI SPECT studies were evaluated visually using the volume rendering method and semi-quantitatively by $3 \mathrm{D}$ subtraction of ${ }^{99 \mathrm{~m}}$ Tc-pertechnetate SPECT from early MIBI SPECT. Of the 61 patients, 40/61 (66 \%) gave positive findings, with solitary lesions detected in 22 patients and more than one lesion in the other 18 , while 16 of these 40 patients (40\%) underwent surgery.

Zhen et al. [44] evaluated the role of MIBI dual-phase planar scanning followed by SPECT/CT in the detection of parathyroid glands for guiding operative therapy. Their series comprised 90 patients with sHPT undergoing hemodialysis for chronic renal disease, all with PTH serum levels $>600 \mathrm{pg} / \mathrm{mL}$. A total of 310 glands were surgically removed after total or subtotal parathyroidectomy. The sensitivity, specificity, and accuracy of SPECT/CT in detecting parathyroid glands were $78.9,100$, and $78.9 \%$, respectively, compared with 55.6, 100, and $55.6 \%$ for planar scanning. The average number of lesions detected on SPECT/CT was significantly higher than the average 



Fig. 1 Patient affected by pHPT with an ectopic mediastinal parathyroid adenoma. Parathyroid dual-tracer $\left({ }^{99} \mathrm{mTc}\right.$-pertechnetate/ MIBI) planar scanning $\mathbf{a}, \mathbf{b}, \mathbf{c}$ revealed a focal area of faint increased uptake of radiotracer in the mediastinum (arrows), more evident in the thorax MIBI view. The focal area was also evident on SPECT $\mathbf{d}$ in the coronal, sagittal, and transverse projections (arrows), but only
SPECT/CT fusion images e with anatomical landmarks were able to precisely define lesion depth and relationship to adjacent structures, localizing the adenoma in the anterosuperior mediastinum, behind the left segment of the manubrium of the sternum and adjacent to the aortic arch (arrows). The exact localization of the ectopic adenoma was confirmed at surgery (color figure online) number detected on planar scanning (3.92 versus 2.84, $P<0.05)$. Moreover, the average longitudinal diameter and average weight of the parathyroids detected by SPECT/CT were significantly smaller than the corresponding values detected by planar scanning $(9.4 \pm 0.7$ versus $14.1 \pm 0.8 \mathrm{~mm}$ and $1,027 \pm 98$ versus $1,321 \pm 104 \mathrm{mg}$, respectively). Furthermore, compared with planar scintigraphy, SPECT/CT also more accurately depicted the precise location of lesions.

The same authors [45] subsequently compared the preoperative diagnostic value of early and delayed MIBI SPECT/CT in a series of 80 patients with the same clinical characteristics as those included in the previous study. The number of lesions detected on early SPECT/CT was similar to the number of lesions evident on delayed studies (3.57 versus 3.55 ), while the specificity value was the same (100\%) for both time points. SPECT/CT showed positive findings only in the early phase in $7 / 80$ cases, while it showed positive findings only in the delayed phase in a further six cases. The authors thus concluded that both early and delayed SPECT/CT should be performed in the preoperative evaluation of hemodialysis patients with sHPT due to chronic kidney disease.

\section{SPECT/CT in patients with previous neck surgery}

In addition to sporadic cases included in some of the studies reported above, selected series of patients with HPT and a history of neck surgery (parathyroidectomy and/or thyroidectomy) have been studied with SPECT/CT. García-Talavera et al. [46] reported a single case of a patient with a neck ectopic parathyroid adenoma that, after several surgical operations and imaging tests had given no significant findings, was finally clearly visualized on early MIBI SPECT/CT in the retropharyngeal space. The adenoma, causing persistent pHPT, was successfully treated. In this specific case, planar scanning was inconclusive, showing a focal area of increased MIBI uptake in the right submandibular region in the early phase which disappeared on delayed imaging. 

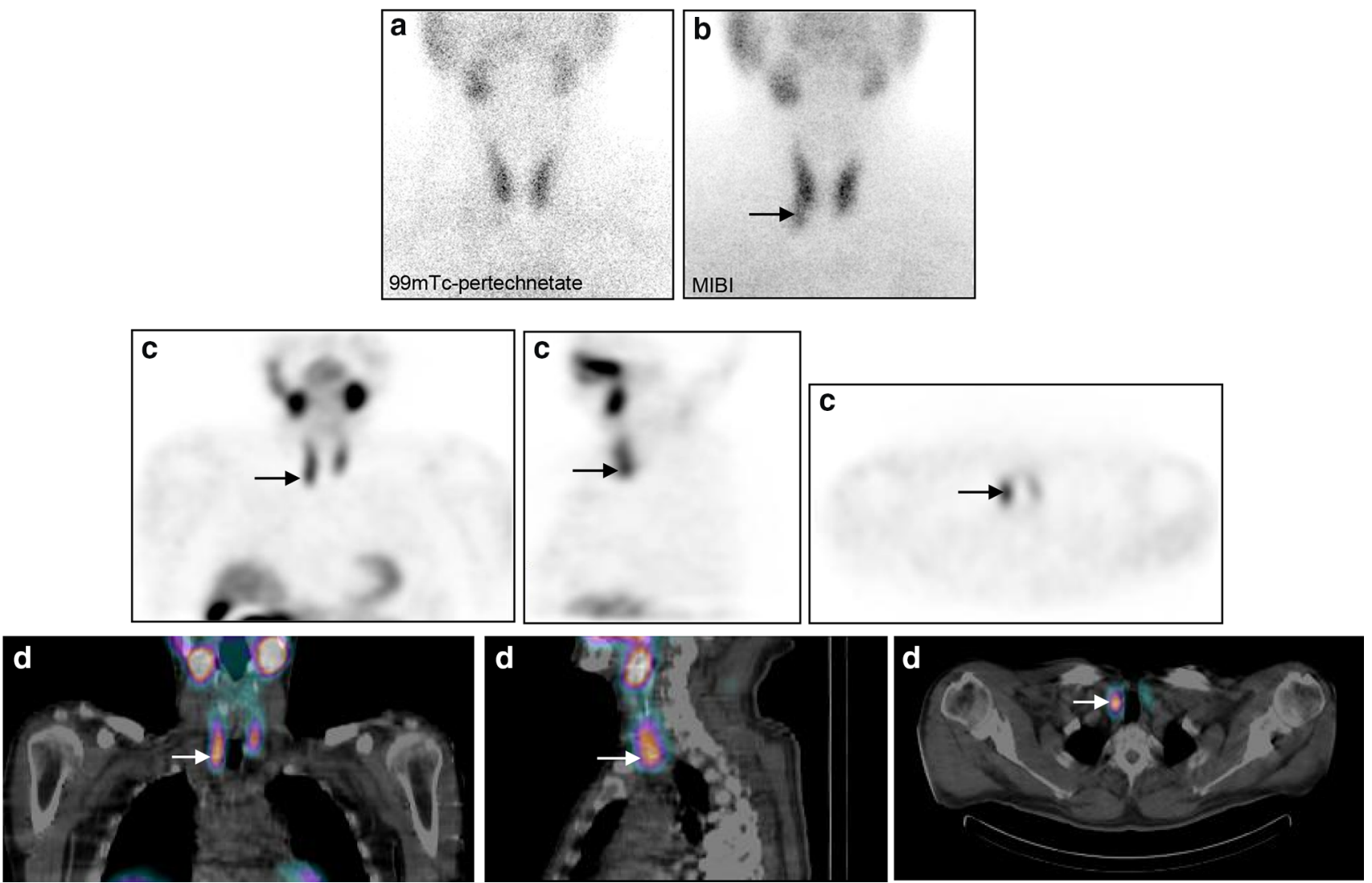

Fig. 2 Patient affected by pHPT with a surgically proven neck parathyroid adenoma. Parathyroid dual-tracer $\left({ }^{99 \mathrm{~m}} \mathrm{Tc}\right.$-pertechnetate/ MIBI) planar scan a, b showed a focal area of increased uptake corresponding to the adenoma (arrow), confirmed by SPECT $\mathbf{c}$ in the coronal, sagittal, and transverse projections (arrows). However, only
SPECT/CT $\mathbf{d}$ fusion images defined the depth of the hyperfunctioning parathyroid gland and added anatomical information that precisely localized it (arrows) in the inferior region of the neck, anteriorly, specifically in the right paratracheal region, below the inferior pole of the right thyroid lobe (inferior parathyroid adenoma) (color figure online)
Another case of persistent pHPT was reported by Gouveia et al. [47], who described a patient with a thoracic ectopic parathyroid adenoma that appeared as a focal area of increased uptake within the mediastinum on dual-phase planar MIBI scan and was precisely localized in the aortopulmonary window on SPECT/CT.

Papathanassiou et al. [48] studied four patients with HPT and prior neck surgery (parathyroidectomy in $2 / 4$ cases and thyroidectomy in $2 / 4$ cases) which had failed to cure the HPT. MIBI SPECT/CT successfully localized the abnormal glands, including an uncommon anterior location of the parathyroid gland and thus allowed efficient surgical treatment, without complications and with a relatively short operation time.

Schalin-Jäntti et al. [49] compared the performance of dual-tracer planar ${ }^{123}$ /MIBI scintigraphy, SPECT/CT, ${ }^{11} \mathrm{C}$ methionine PET/CT, and selective venous sampling (SVS) in a series of 21 patients with pHPT referred for reoperation because of persistent disease. Eighteen $(86 \%)$ of the 21 patients were biochemically cured after the operation. Nineteen parathyroid glands (9 adenomas, 1 atypical adenoma, and 9 hyperplastic glands) were removed from 17/18 patients, while the histology results in the remaining patient, who was biochemically cured, were unclear. The accuracy in localizing a pathological parathyroid gland to the correct side of the neck was $59 \%$ for planar scanning, $19 \%$ for SPECT/CT, $65 \%$ for PET/CT, and $40 \%$ for SVS. In the $3 / 21$ patients who were not cured, preoperative planar scanning and SPECT/CT continued to give negative results, SVS was false negative in all of them and ${ }^{11} \mathrm{C}$ methionine PET/CT in one. PET/CT accurately revealed the pathological gland in 4/8 $(50 \%)$ patients with negative planar scintigraphy, and all of these were biochemically cured after reoperation. The authors concluded that ${ }^{123} \mathrm{I} /$ MIBI planar scanning performs well in persistent HPT patients and that it is recommended as the first-line imaging procedure before reoperation. ${ }^{11} \mathrm{C}$-methionine PET/CT provides valuable additional information if ${ }^{123} \mathrm{I} /{ }^{99 \mathrm{~m}} \mathrm{Tc}$-sestaMIBI planar scans remain negative, whereas SPECT/CT and SVS provide no additional information compared with the combined results of ${ }^{123} \mathrm{I} / \mathrm{MIBI}$ planar scintigraphy and ${ }^{11} \mathrm{C}$-methionine PET/CT. 

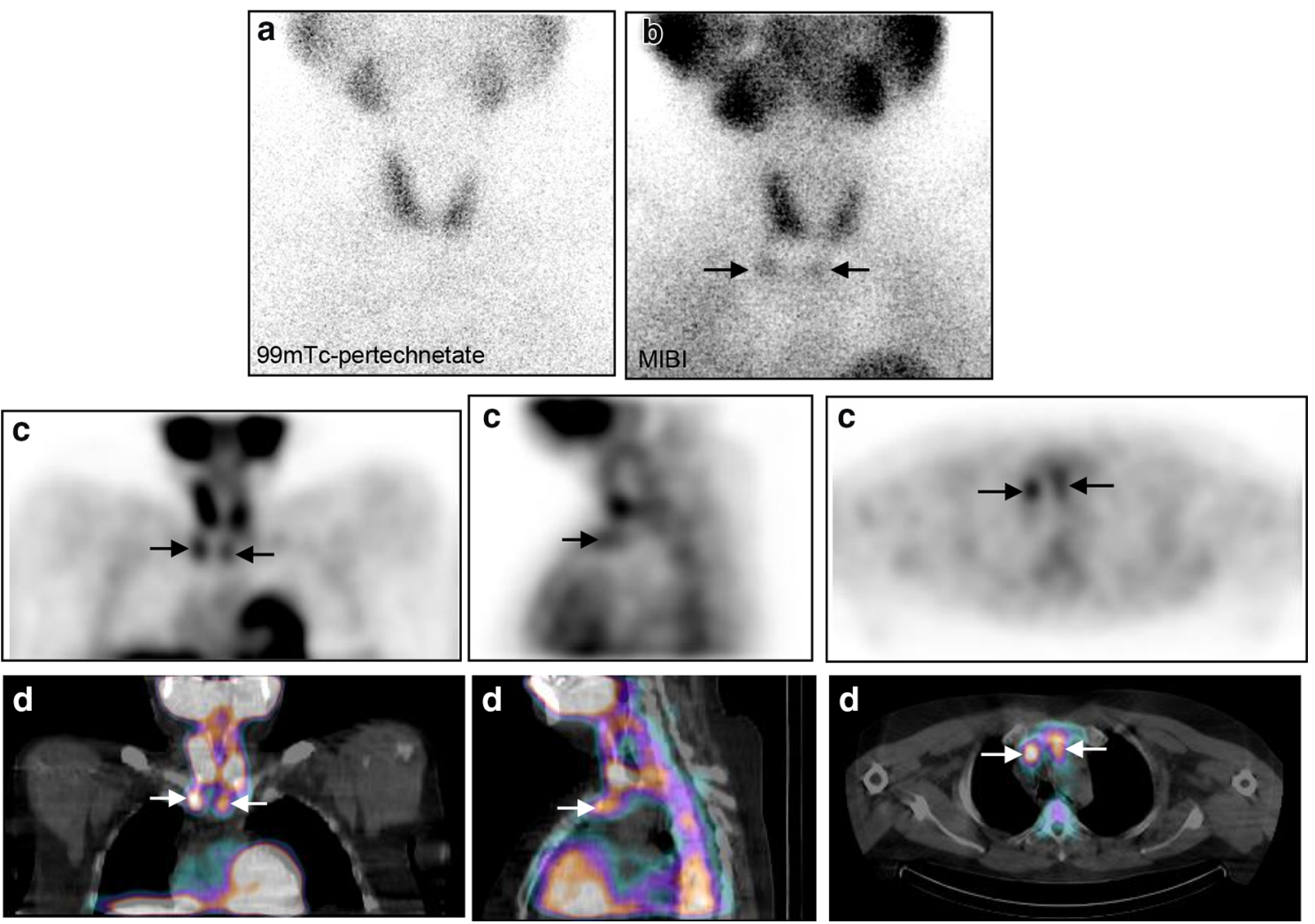

Fig. 3 Patient affected by sHPT with recurrent disease already submitted to parathyroidectomy. Parathyroid dual-tracer $\left({ }^{99 \mathrm{~m}} \mathrm{Tc}\right.$ pertechnetate/MIBI) planar scanning a, b revealed two focal areas of increased uptake in the neck below the inferior poles of the thyroid gland (arrows). SPECT c, in coronal, sagittal, and transverse

Wimmer et al. [50] prospectively studied 28 consecutive patients, 21 with previous thyroidectomy and seven with previous parathyroidectomy, comparing MIBI SPECT/CT with SPECT alone. Image fusion was able to predict the exact position of the abnormal gland in $24 / 28$ patients (86 \%), whereas SPECT did so in only $12 / 28$ cases $(43 \%$, $P<0.004)$. SPECT/CT detected all three pathological glands located in an ectopic position, whereas SPECT detected only one of them. The specificity was $97 \%$ for SPECT/CT and $92 \%$ for SPECT alone, while the overall accuracy was $95 \%$ for SPECT/CT and $81 \%$ for SPECT. Differently to all the other studies cited above, the CT component employed in this latter study was highly diagnostic since it was acquired after infusion of contrast medium. CT and SPECT datasets were acquired separately and then fused by means of special image fusion software allowing a "virtual neck exploration" by showing the suspected gland three-dimensionally in relationship to anatomical landmarks. The same authors also used this acquisition protocol in selected series of patients with single-gland disease or MGD at their first surgical exploration [51, 52], comparing SPECT/CT image fusion with $\mathrm{CT}$ and SPECT alone. These studies provided evidence that projections, better defined the two areas (arrows), but only SPECT/ CT d fusion images with anatomical landmarks precisely localized the lesions behind the right sternoclavicular and the left parasternal regions, respectively, where two ectopic hyperplastic glands mixed with thymic tissue were found at surgery (color figure online)

SPECT/CT is superior to the other two diagnostic procedures for the preoperative localization of enlarged parathyroid glands.

\section{Summary}

Hybrid SPECT/CT imaging is playing an increasingly important role in nuclear medicine in several oncological and non-oncological fields as it can improve the diagnostic accuracy of conventional scintigraphic techniques and impact on patient management [53-57]. This paper has focused on the clinical role of SPECT/CT in patients with HPT, highlighting the certainties and the controversies emerging from a review of the literature dealing with this specific setting. SPECT/CT is clearly superior to both conventional planar parathyroid scintigraphy and SPECT for the anatomical localization of abnormal parathyroid glands and thus facilitates their surgical treatment. This fact has considerable implications for patients with primary HPT scheduled to undergo minimally invasive parathyroidectomy. The HPT patients found to benefit most from SPECT/CT are those with ectopic adenomas, mainly in the 
mediastinum, and those with previous neck surgery. In some studies, SPECT/CT has also demonstrated a higher sensitivity than that of conventional imaging methods, especially in the detection of low-weight parathyroid adenomas and in the presence of concomitant nodular goiter. In other studies, SPECT/CT also proved more specific, with anatomical CT data helping in the correct categorization of MIBI-avid non-parathyroid lesions as thyroid nodules or artifacts. No definitive conclusions on the clinical value of SPECT/CT in patients with secondary HPT and MGD can be drawn due to the small number of studies thus far conducted in this specific context, but the current available data nevertheless seem to suggest that fusion imaging is able to detect a significantly higher number of hyperplastic glands than planar scanning can. Considering the relatively high rate of first surgery failure in patients with SHPT, the employment of preoperative parathyroid scintigraphy in combination with SPECT/CT should probably be considered not only in cases with previous neck surgery, but also in those at their first surgical operation to increase the possibility of localizing ectopic or supernumerary glands, allowing the surgeon to adopt a selective surgical approach and reduce surgical failure.

SPECT/CT has been included in dual-tracer and in single-tracer dual-phase parathyroid scintigraphic protocols, in the latter protocol in the early phase, the delayed phase, or both. There is no certainty regarding the superiority of one methodology over the other (single versus dual-tracer protocol). However, in the single-tracer protocol, dual-time acquisition seems to perform better than single-time acquisition, especially when combining early SPECT/CT with any delayed method (SPECT/CT, SPECT, or planar imaging) $[30,31]$. Thus, in the dual-phase protocol, to limit patient exposure from the CT component, SPECT/CT can be acquired only at a single time point, preferably the early phase. On the other hand, a low-dose $\mathrm{CT}$, such as that employed in the majority of studies reported in the literature, with estimated CT doses ranging from 0.1 to $2 \mathrm{mSv}$ according to the volume scanned (limited to the neck or including both neck and thorax) and the device used for image acquisition, appears sufficient for the anatomical depiction of SPECT lesions and for attenuation correction [58].

It can be concluded, on the basis of the literature data, that SPECT/CT plays an important role in the preoperative localization of hyperfunctioning parathyroid glands. Thus, it should be included in parathyroid scintigraphy protocols as a complementary tool to conventional planar scanning.

Conflict of interest Angela Spanu, Orazio Schillaci, Bastiana Piras, and Giuseppe Madeddu declare that they have no conflicts of interest.
Human and animal studies This article does not contain any studies with human or animal subjects performed by any of the authors.

\section{References}

1. Fraser WD (2009) Hyperparathyroidism. Lancet 374:145-158

2. Felger EA, Kandil E (2010) Primary hyperparathyroidism. Otolaryngol Clin North Am 43:417-432

3. Jamal SA, Miller PD (2013) Secondary and tertiary hyperparathyroidism. J Clin Densitom 16:64-68

4. Alhefdhi A, Schneider DF, Sippel R, Chen H (2014) Recurrent and persistence primary hyperparathyroidism occurs more frequently in patients with double adenomas. J Surg Res 190:198-202

5. Taïeb D, Ureña-Torres $P$, Zanotti-Fregonara P, Rubello D, Ferretti A, Henter I, Henry JF, Schiavi F, Opocher G, Blickman JG, Colletti PM, Hindié E (2013) Parathyroid scintigraphy in renal hyperparathyroidism: the added diagnostic value of SPECT and SPECT/CT. Clin Nucl Med 38:630-635

6. Hindié E, Ugur O, Fuster D, O’Doherty M, Grassetto G, Urena P, Kettle A, Gulec SA, Pons F, Rubello D (2009) 2009 EANM Parathyroid guidelines. Eur J Nucl Med Mol Imaging 36:1201-1216

7. Greenspan BS, Dillehay G, Intenzo C, Lavely WC, O'Doherty M, Palestro CJ, Scheve W, Stabin MG, Sylvestros D, Tulchinsky M (2012) SNM practice guideline for parathyroid scintigraphy 4.0. J Nucl Med Technol 40:111-118

8. Ogilvie CM, Brown PL, Matson M, Dacie J, Reznek RH, Britton K, Berney D, Drake WM, Jenkins PJ, Chew SL, Monson JP (2006) Selective parathyroid venous sampling in patients with complicated hyperparathyroidism. Eur J Endocrinol 155:813-821

9. Spanu A, Schillaci O, Madeddu G (2005) ${ }^{99 \mathrm{~m}} \mathrm{Tc}$ labelled cationic lipophilic complexes in malignant and benign tumors: the role of SPET and pinhole-SPET in breast cancer, differentiated thyroid carcinoma and hyperparathyroidism. Q J Nucl Med Mol Imaging 49:145-169

10. Perez-Monte JE, Brown ML, Shah AN, Ranger NT, Watson CG, Carty SE, Clarke MR (1996) Parathyroid adenomas: accurate detection and localization with Tc-99 m sestamibi SPET. Radiology 201:85-91

11. Moka D, Voth E, Dietlein M, Larena-Avellaneda A, Schicha H (2000) Technetium ${ }^{99 m}$-MIBI-SPET: a highly sensitive diagnostic tool for localization of parathyroid adenomas. Surgery 128:29-35

12. Lorberboym M, Minski I, Macadziob S, Nikolov G, Schachter P (2003) Incremental diagnostic value of preoperative ${ }^{99 \mathrm{~m}} \mathrm{Tc}-\mathrm{MIBI}$ SPECT in patients with a parathyroid adenoma. J Nucl Med 44:904-908

13. Gallowitsch HJ, Mikosch P, Kresnik E, Unterweger O, Lind P (2000) Comparison between ${ }^{99 \mathrm{~m}} \mathrm{Tc}$-tetrofosmin/pertechnetate subtraction scintigraphy and ${ }^{99 \mathrm{~m}} \mathrm{Tc}$-tetrofosmin SPECT for preoperative localization of parathyroid adenoma in an endemic goiter area. Invest Radiol 35:453-459

14. Staudenherz A, Abela C, Niederle B, Steiner E, Helbich T, Puig S, Kaserer K, Becherer A, Leitha T, Kletter K (1997) Comparison and histopathological correlation of three parathyroid imaging methods in a population with a high prevalence of concomitant thyroid diseases. Eur J Nucl Med 24:143-149

15. Billotey C, Sarfati E, Aurengo A, Duet M, Mundler O, Toubert ME, Rain JD, Najean Y (1996) Advantages of SPECT in technetium-99 m-sestamibi parathyroid scintigraphy. J Nucl Med 37:1773-1778

16. Civelek AC, Ozalp E, Donovan P, Udelsman R (2002) Prospective evaluation of delayed technetium $-99 \mathrm{~m}$ sestamibi SPECT scintigraphy for preoperative localization of primary hyperparathyroidism. Surgery 131:149-157 
17. Gallowitsch HJ, Mikosch P, Kresnik E, Gomez I, Lind P (1997) Technetium $99 \mathrm{~m}$ tetrofosmin parathyroid imaging results with double-phase study and SPECT in primary and secondary hyperparathyroidism. Invest Radiol 32:459-465

18. Neumann DR, Esselstyn CB Jr, Madera A, Wong CO, Lieber M (1998) Parathyroid detection in secondary hyperparathyroidism with ${ }^{123} \mathrm{I} /{ }^{99 \mathrm{~m}} \mathrm{Tc}$-sestamibi subtraction single photon emission computed tomography. J Clin Endocrinol Metab 83:3867-3871

19. Neumann DR, Esselstyn CB Jr, Madera AM (2000) Sestamibi/ iodine subtraction single photon emission computed tomography in reoperative secondary hyperparathyroidism. Surgery 128:22-28

20. Spanu A, Migaleddu V, Manca A, Falchi A, Marongiu P, Pisu N, Chessa F, Madeddu G (2003) The usefulness of single photon emission computerized tomography with pinhole collimator (PSPECT) in preoperative localization of hyperfunctioning parathyroid glands in patients with secondary hyperparathyroidism. Radiol Med 106:399-412

21. Spanu A, Falchi A, Manca A, Marongiu P, Cossu A, Pisu N, Chessa F, Nuvoli S, Madeddu G (2004) The usefulness of neck pinhole SPECT as a complementary tool to planar scintigraphy in primary and secondary hyperparathyroidism. J Nucl Med 45:40-48

22. Ruf J, Seehofer D, Denecke D, Steketer L, Rayes N, Felix R, Amthauer H (2007) Impact of image fusion and attenuation correction by SPECT-CT on the scintigraphic detection of parathyroid adenomas. Nuklearmedizin 46:15-21

23. Ng P, Lenzo NP, McCarthy MC, Thompson I, Leedman PJ (2003) Ectopic parathyroid adenoma localised with sestamibi SPECT and image-fused computed tomography. Med J Aust 179:485-487

24. Rubello D, Casara D, Fiore D, Muzzio P, Zonzin G, Shapito B (2002) An ectopic mediastinal parathyroid adenoma accurately located by a single-day imaging protocol of Tc- $99 \mathrm{~m}$ pertechnetate-MIBI subtraction scintigraphy and MIBI-SPECT-computed tomographic image fusion. Clin Nucl Med 27:186-190

25. Kaczirek K, Prager G, Kienast O, Dobrozemsky G, Dudczak R, Niederle B, Kurtaran A (2003) Combined transmission and ${ }^{(99 m)}$ Tc-sestamibi emission tomography for localization of mediastinal parathyroid glands. Nuklearmedizin 42:220-223

26. Gayed IW, Kim EE, Broussard WF, Evans D, Lee J, Broemeling LD, Ochoa BB, Moxley DM, Erwin WD, Podoloff DA (2005) The value of ${ }^{99 m}$ Tc-sestamibi SPECT/CT over conventional SPECT in the evaluation of parathyroid adenomas or hyperplasia. J Nucl Med 46:248-252

27. Serra A, Bolasco P, Satta L, Nicolosi A, Uccheddu A, Piga M (2006) Role of SPECT/CT in the preoperative assessment of hyperparathyroid patients. Radiol Med 111:999-1008

28. Krausz Y, Bettman L, Guralnik L, Yosilevsky G, Keidar Z, BarShalom R, Even-Sapir E, Chisin R, Israel O (2006) Technetium- ${ }^{99 \mathrm{~m}}$-MIBI SPECT/CT in primary hyperparathyroidism. World J Surg 30:76-83

29. Harris L, Yoo J, Driedger A, Fung K, Franklin J, Gray D, Holliday R (2008) Accuracy of technetium-99m SPECT-CT hybrid images in predicting the precise intraoperative anatomical location of parathyroid adenomas. Head Neck 30:509-517

30. Lavely WC, Goetze S, Friedman KP, Leal JP, Zhang Z, GarretMayer E, Dackiw AP, Tufano RP, Zeiger MA, Ziessman HA (2007) Comparison of SPECT/CT, SPECT, and planar imaging with single- and dual-phase ${ }^{99 \mathrm{~m}} \mathrm{Tc}$-sestamibi parathyroid scintigraphy. J Nucl Med 48:1084-1089

31. Tokmak H, Demirkol MO, Alagöl F, Tezelman S, Terzioglu T (2014) Clinical impact of SPECT-CT in the diagnosis and surgical management of hyper-parathyroidism. Int $\mathrm{J}$ Exp Med 7:1028-1034
32. Öksüz MÖ, Dittmann H, Wicke C, Müssig K, Bares R, Pfannenberg C, Eschmann SM (2011) Accuracy of parathyroid imaging: a comparison of planar scintigraphy, SPECT, SPECT$\mathrm{CT}$, and C-11 methionine PET for the detection of parathyroid adenomas and glandular hyperplasia. Diagn Interv Radiol 17:297-307

33. Ciappuccini R, Morera J, Pascal P, Rame JP, Heutte N, Aide N, Babin E, Reznik YM, Bardet S (2012) Dual-phase ${ }^{99 \mathrm{~m}} \mathrm{Tc}$ sestamibi scintigraphy with neck and thorax SPECT/CT in primary hyperparathyroidism. Clin Nucl Med 37:223-228

34. Bural GG, Muthukrishnan A, Oborski MJ, Mountz JM (2012) Improved benefit of SPECT/CT compared to SPECT alone for the accurate localization of endocrine and neuroendocrine tumors. Mol Imaging Radionucl Ther 21:91-96

35. Pata G, Casella C, Besuzio S, Mittempergher F, Salerni B (2010) Clinical appraisal of $99 \mathrm{~m}$ technetium-sestamibi SPECT/CT compared to conventional SPECT in patients with primary hyperparathyroidism and concomitant nodular goiter. Thyroid 20:1121-1127

36. Shafiei B, Hoseinzadeh S, Fotouhi F, Malek H, Azizi F, Jahed A, Hadaegh F, Salehian M, Parsa H, Javadi H, Assadi M (2012) Preoperative ${ }^{99 \mathrm{~m}} \mathrm{Tc}$-sestamibi scintigraphy in patients with primary hyperparathyroidism and concomitant nodular goiter: comparison of SPECT-CT, SPECT, and planar imaging. Nucl Med Commun 33:1070-1076

37. Patel CN, Salahudeen HM, Lansdown M, Scarsbrook AF (2010) Clinical utility of ultrasound and ${ }^{99 \mathrm{~m}} \mathrm{Tc}$ sestamibi SPECT/CT for preoperative localization of parathyroid adenoma in patients with primary hyperparathyroidism. Clin Radiol 65:278-287

38. Noda S, Onoda N, Kashiwagi S, Kawajiri H, Takashima T, Ishikawa T, Yoshida A, Higashiyama S, Kawabe J, Imanishi Y, Tahara H, Inaba M, Osawa M, Hirakawa K (2014) Strategy of operative treatment of hyperparathyroidism using US scan and ${ }^{(99 \mathrm{~m})}$ Tc-MIBI SPECT/CT. Endocr J 61:225-230

39. Kim YI, Jung YH, Hwang KT, Lee HY (2012) Efficacy of ${ }^{99 m} \mathrm{Tc}$ sestamibi SPECT/CT for minimally invasive parathyroidectomy: comparative study with ${ }^{99 \mathrm{~m}} \mathrm{Tc}$-sestamibi scintigraphy, SPECT, US and CT. Ann Nucl Med 26:804-810

40. Neumann DR, Obuchowski NA, Difilippo FP (2008) Preoperative ${ }^{123} \mathrm{I} /{ }^{99 \mathrm{~m}} \mathrm{Tc}$-sestamibi subtraction SPECT and SPECT/CT in primary hyperparathyroidism. J Nucl Med 492:2012-2017

41. Hassler S, Ben-Sellem D, Hubele F, Constantinesco A, Goetz C (2014) Dual-isotope ${ }^{99 \mathrm{~m}} \mathrm{Tc}-\mathrm{MIBI} /{ }^{123} \mathrm{I}$ parathyroid scintigraphy in primary hyperparathyroidism: comparison of subtraction SPECT/ CT and pinhole planar scan. Clin Nucl Med 39:32-36

42. Cytawa W, Teodorczyk J, Lass P (2013) Advantages of hybrid SPECT-CT imaging in preoperative localization of parathyroid glands in a patients with secondary hyperparathyroidism. A case report. Pol J Radiol 78:81-84

43. Croustowa D, Kubinyi J, Trnka J, Adamek S (2014) The role of ${ }^{99 \mathrm{~m}}$ Tc-MIBI SPECT/low dose CT with 3D subtraction in patients with secondary hyperparathyroidism due to chronic kidney disease. Endocr Regul 48:55-63

44. Zhen L, Li H, Liu X, Ge BH, Yan J, Yang J (2013) The application of SPECT/CT for preoperative planning in patients with secondary hyperparathyroidism. Nucl Med Commun 34:439-444

45. Yang J, Hao R, Yuan L, Li C, Yan J, Zhen L (2014) Value of dual-phase $(99 \mathrm{~m}) \mathrm{Tc}$-sestamibi scintigraphy with neck and thoracic SPECT/CT in secondary hyperparathyroidism. AJR Am J Roentgenol 202:180-184

46. García-Talavera P, González ML, Aís G, Olmos R, Ruis MA, Sainz A, Gamazo C (2012) SPECT-CT in the localization of an ectopic retropharyngeal parathyroid adenoma as a cause for persistent primary hyperparathyroidism. Rev Esp Med Nucl Imagen Mol 31:275-277 
47. Gouveia S, Rodrigues D, Barros L, Ribeiro C, Albuquerque A, Costa G, Carvalheiro M (2012) Persistent primary hyperparathyroidism: an uncommon location for an ectopic gland-case report and review. Arq Bras Endocrinol Metab 56:393-403

48. Papathanassiou D, Flament JB, Pochart JM, Patey M, Marty H, Liehn JC, Schvartz C (2008) SPECT/CT in localization of parathyroid adenoma or hyperplasia in patients with previous neck surgery. Clin Nucl Med 33:394-397

49. Schalin-Jäntti C, Ryhänen E, Heiskanen I, Seppänen M, Arola J, Schildt J, Väisänen M, Nelimarkka L, Lisinen I, Aalto V, Nuutila P, Välimäki MJ (2013) Planar scintigraphy with ${ }^{123} \mathrm{I}^{99 \mathrm{~m}} \mathrm{Tc}$-sestamibi, ${ }^{99 \mathrm{~m}} \mathrm{Tc}$-sestamibi SPECT/CT, ${ }^{11} \mathrm{C}$-methionine PET/CT, or selective venous sampling before reoperation of primary hyperparathyroidism? J Nucl Med 54:739-747

50. Wimmer G, Bale R, Kovacs P, Gabriel M, Putzer D, Sauper T, Sieb M, Profanter C, Margreiter R, Prommegger R (2008) Virtual neck exploration in patients with hyperparathyroidism and former cervical operations. Langenbecks Arch Surg 393:687-692

51. Prommegger R, Wimmer G, Profanter C, Sauper T, Sieb M, Kovacs P, Bale R, Putzer D, Gabriel M, Margreiter R (2009) Virtual neck exploration. A new method for localizing abnormal parathyroid glands. Ann Surg 250:761-765

52. Wimmer G, Profanter C, Kovacs P, Sieb M, Gabriel M, Putzer D, Margreiter R, Prommegger R (2010) CT-MIBI-SPECT image fusion predicts multiglandular disease in hyperparathyroidism. Langenbecks Arch Surg 395:73-80

53. Spanu A, Solinas ME, Chessa F, Sanna D, Nuvoli S, Madeddu G (2009) ${ }^{131}$ I SPECT/CT in the follow-up of differentiated thyroid carcinoma: incremental value versus planar imaging. J Nucl Med 50:184-190

54. Spanu A, Chessa F, Sanna D, Cottu P, Manca A, Nuvoli S, Madeddu G (2009) Scintimammography with a high resolution dedicated breast camera in comparison with SPECT/CT in primary breast cancer detection. Q J Nucl Med Mol Imaging 53:271-280

55. Schillaci O, Spanu A, Tagliabue L, Filippi L, Danieli R, Palumbo B, Del Sole A, Madeddu G (2009) SPECT/CT with a hybrid imaging system in the study of lower gastrointestinal bleeding with technetium-99m red blood cells. Q J Nucl Med Mol Imaging 53:281-289

56. Filippi L, Uccioli L, Giurato L, Schillaci O (2009) Diabetic foot infection: usefulness of SPECT/CT for ${ }^{99 \mathrm{~m}} \mathrm{Tc}-\mathrm{HMPAO}$-labeled leukocyte imaging. J Nucl Med 50:1042-1046

57. Abikhzer G, Keidar Z (2014) SPECT/CT and tumor imaging. Eur J Nucl Med Mol Imaging 41(Suppl 1):S67-S80

58. Buck A, Nekolla S, Ziegler S, Beer A, Krause BJ, Herrmann K, Scheidhauer K, Wester HJ, Rummeny EJ, Schwaiger M, Drzezga A (2008) SPECT/CT. J Nucl Med 49:1305-1319 